\title{
Relational Learning for Spatial Relation Extraction from Natural Language
}

\author{
Parisa Kordjamshidi ${ }^{1}$, Paolo Frasconi ${ }^{2}$, Martijn Van Otterlo ${ }^{3}$, \\ Marie-Francine Moens ${ }^{1}$, and Luc De Raedt ${ }^{1}$ \\ ${ }^{1}$ Department of Computer Science, Katholieke Universiteit Leuven, Belgium \\ ${ }^{2}$ Università degli Studi di Firenze, Italy \\ 3 Radboud University Nijmegen, The Netherlands
}

\begin{abstract}
Automatically extracting spatial information is a challenging novel task with many applications. We formalize it as an information extraction step required for a mapping from natural language to formal spatial representation. Sentences may give rise to multiple spatial relations between words representing landmarks, trajectors and spatial indicators. Our contribution is to formulate the extraction task as a relational learning problem, for which we employ the recently introduced $\mathrm{kLog}$ framework. We discuss representational and modeling aspects, kLog's flexibility in our task and we present current experimental results.
\end{abstract}

\section{Introduction}

An essential function of language is to express spatial relationships between objects and their relative location in space. Understanding linguistic spatial descriptions is a challenging problem in robotics, navigation, human-machine interaction, query answering systems, cf. [18], and automated extraction of spatial information from natural language is of particular interest.

Here we provide a relational learning representation and solution to the so called spatial role labeling (SpRL) problem. This problem was recently introduced [14] and is concerned with the extraction of generic spatial semantics from natural language. The core problem of SpRL is: i) the identification of the words that play a role in describing spatial concepts, and ii) the classification of the role that these words play in the spatial configuration. The main roles are:

Trajector. The entity, i.e., person, object or event whose location is described, which can be static or dynamic; (also called: local/figure object, locatum).

Landmark. The reference entity in relation to which the location or the motion of the trajector is specified. (also called: reference object or relatum).

Spatial indicator. The element that defines constraints on spatial properties such as the location of the trajector with respect to the landmark. The spatial indicator determines the type of spatial relation.

Spatial indicators are often prepositions, particularly in English language, although these can be a verb, noun, adjective, or adverb too. However, the sense 
depends on the context. For example, in the sentence "Give me the book on AI on the big table behind the wall.", the second preposition on is an indicator of a spatial relation between book and table, while the first preposition on has no spatial sense but states the topic of the book. In this sentence, book is the trajector of the on-relation and the phrase the table is the landmark. The spatial relations that can be extracted from the sentence are on (book, table), behind (table, wall). From this one can infer that also the statement behind (book, wall) holds using spatial reasoning $[21,14]$. Note that on (book, AI) is not a spatial relation.

We identify two main abstraction layers for the extraction of spatial information [1,13,12]: 1) a linguistic layer, which starts with unrestricted natural language and predicts the existence of spatial information at the sentence level by identifying the words that play a particular spatial role as well as their spatial relationship; 2) a formal layer, in which the spatial roles are mapped onto a spatial calculus model [9]. In the above mentioned example a first step is to identify that the spatial relation (on) holds between book and table. In the second step, this relation could be mapped to a specific, formal spatial representation, e.g., AboveExternallyConnected(book, table). In particular, in terms of the RCC (Regional Connection Calculus) [3] the relation is presented as an EC (externally connected) relationship between book and table. In practice, in the context of the extraction of spatial relation from natural language, a few related works exist that extract specific and application-dependent relations from text using restricted languages. Our recent work [15] provides an extensive review of the state-of-the-art and systematically investigates spatial relation and role extraction from unrestricted natural language. In those experiments conditional random fields $(\mathrm{CRF})$ have been employed. Moreover we have investigated the automatic mapping from natural language to spatial calculus representations in $[13,12]$.

In the present paper we focus on the first (linguistic) level which is a necessary step for mapping natural language to any formal spatial calculus. The key contribution of this work is that we employ an expressive logical representation and relational model of SpRL. We utilize kLog [8], a novel framework for kernel-based relational learning that uses graph kernels. It supports describing learning problems in a declarative, relational fashion. Typical for relational learning settings [6], kLog is able to exploit background knowledge in the form of logic programs, which also enables reasoning with the extracted spatial relations for spatial understanding. This work is towards our long-term research goal to develop an integrated framework for spatial learning and reasoning based on natural language descriptions.

To extract the spatial relations, we use two learning settings in this paper. One is a binary classification setting where the examples are a set of positive and negative spatial relations produced from the original sentences using a set of rules. The second setting is a sequence tagging approach where the words in a sentence are tagged by a set of spatial roles. After the sequence tagging step, the spatial relations are produced using a basic set of rules. The results of the sequence tagging setting are outperforming the first setting and are com- 
parable to CRFs. Using kLog's language, the structure of the input and output is represented relationally and the propositionalization is done automatically. Hence kLog is more expressive and flexible in the modeling of the problem and performing relational feature engineering.

The paper is structured as follows. In Section 2, spatial role labeling is defined formally, kLog is briefly introduced, and representation and features are defined. In Section 3 we discuss two ways for modeling the learning problem: as triplet classification (i.e. hyperlink prediction) and sequence tagging. Section 4 reports on experimental results that show the promise of our approach, and finally Section 5 concludes and points out future directions.

\section{Relational Learning for SpRL}

Many natural language processing problems, including SpRL, require one to deal with the underlying structure of the data, to employ knowledge about the domain such as ontologies, and to impose constraints on outcomes. Therefore, a relational formulation of SpRL is encouraged and many related problems can be treated as such [5]. Below we formulate the SpRL problem in the kLog framework.

\subsection{Problem statement}

We formulate SpRL as supervised learning. The input is a natural language sentence $S$ that is a sequence of $M$ words $S=\left\langle w_{1}, w_{2}, \ldots, w_{M}\right\rangle$. The words have a number of properties and relationships. A spatial relation is presented as a predicate with three arguments sr (SI, TR, LM). Three spatial role predicates are defined as indicator(SI), trajector (TR), landmark(LM) where $\mathrm{SI}=w_{i}$, $\mathrm{TR}=w_{j}, \mathrm{LM}=w_{k}, i, j, k \in[1, M]$ and for each spatial triplet $i \neq j \neq k$. Each sentence contains $n \geq 0$ spatial relations. Each word is an argument of the spatial role predicates when it carries a specific spatial role. For any spatial relation, when no word in the sentence $S$ represents a trajector or a landmark, then the value of those arguments is "undefined". In those cases, the trajector or landmark is implicit. In general, spatial indicators, trajectors and landmarks can be arbitrary segments that contain more than one word, but here we focus on individual words; namely the syntactical head word of a segment. This is a common simplifying assumption in similar tasks such as semantic role labeling that could be extended to constituent based segmentation or BIO tagging in the future [17]. For example, when a phrase such as "the huge blue book" is a trajector then only the so-called head word of this phrase "book" is considered as the trajector and the other words have no role. As an illustration, consider the following examples.

A girl and a boy are sitting at the desk in the classroom.

The spatial relations are: sr (at, girl, desk), sr (at, boy, desk). Depending on the learning model these spatial roles can be predicted as intermediate steps,

trajector(girl) trajector(boy) landmark(desk) landmark(classroom). 


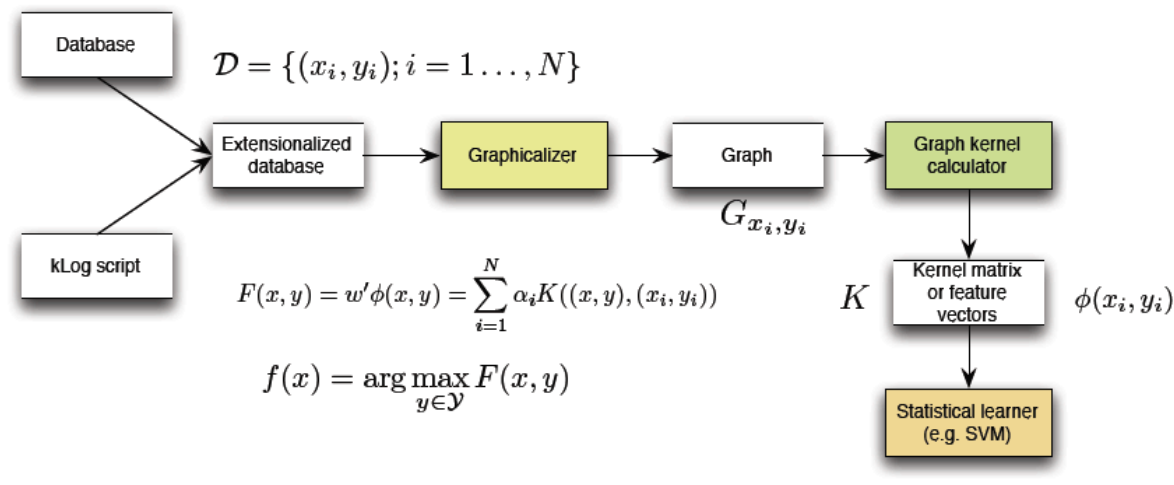

Fig. 1. kLog flow

An example with an implicit trajector is the following sentence,

Go under the bridge.

In this sentence the target spatial relation is: sr (under, undefined,bridge) and the spatial roles are indicator (under) and landmark (bridge).

Before describing the relational input features and modeling the problem, the kLog framework and language is discussed in the following subsection.

\subsection{Relational representation and learning in $\mathrm{kLog}$}

$\mathrm{kLog}[8]^{4}$ is a language designed for relational learning. $\mathrm{kLog}$ allows users to specify a relational database, background knowledge and the target problem itself in a declarative way. The main elements of the data model are entities and relationships. kLog's signatures represent the format of each table in a relational database. This is to some extent similar to the notion of bias in inductive logic programming. In this way the data's structure is naturally presented as an entityrelationship $(\mathrm{E} / \mathrm{R})$ diagram like the one shown in Fig. 2(a).

The purpose of $\mathrm{kLog}$ is to make it easy to define and maintain relational features. $\mathrm{kLog}$ is a domain-specific language embedded in Prolog, a language with both a declarative and a procedural semantics. Hence, feature definition in kLog lies somewhat between purely declarative (as in Markov logic [7]) and purely imperative (as in FACTORIE [16]) approaches.

$\mathrm{kLog}$ learns from interpretations [6], where an interpretation is essentially a set of ground atoms (or a set of tuples, since structured terms are not allowed in the language). Ground atoms can be either explicitly given as data (for extensional signatures) or deduced using Prolog's deduction mechanism (for intensional signatures). Intensional predicates are akin to Datalog rules [10] and tabling can also be used to avoid Prolog's procedural semantics, if desired. Under mild assumptions, grounding the E/R diagram (a process we call graphicalization) yields, for each interpretation, a bipartite graph whose nodes are ground

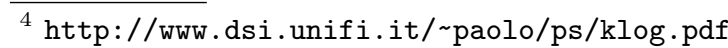


atoms either of entity or relationship type, cf. Fig. 2(b). An edge from a relationship vertex to an entity vertex is created iff the identifier of the entity appears as an argument of the ground relationship atom. A graph kernel is subsequently used to compare interpretations by comparing the associated graphs. Unlike the approach in [20], kLog does not require a kernel on hypergraphs and any existing graph kernel can - in principle - be used. The current implementation uses a modified version of the neighborhood subgraph pairwise distance kernel (NSPDK) [4]. In practice, NSPKD is more efficiently computed by creating feature vectors associated with interpretations. Any model can then be trained using the feature vectors, Fig. 1 presents the workflow in kLog. kLog is also agnostic about the statistical procedure used to learn from the constructed feature vectors and several alternative models can be plugged-in. In this work, the underlying models are a binary SVM and SVM-HMM which is briefly described.

SVM-HMM This model is used in conjunction with kLog in the current experiments. SVM-HMM and linear-chain CRF are extensions of SVM and the logistic regression model, respectively, for sequence tagging. They can be constructed to share exactly the same feature space and both use a linear model as their decision function: $f(x)=\arg \max _{y} w^{\top} \cdot \phi(x, y)$, where $\phi(x, y)$ is the feature vector associated with each interpretation, $(x, y)$, consisting of input $(x)$ and output $(y)$ ground atoms. A potential function equal to $w^{\top} \cdot \phi(x, y)$ is used to score the interpretation. Prediction, is the process of maximizing $F$ with respect to $y$. Learning is the process of fitting $w$ to the training data and this differs between the two models. Both CRFs and SVM-HMM models may be extended to model arbitrary structures. For example Markov logic [7] provides a relational language to represent Markov random field models with any arbitrary structure. On the other hand, kLog is a declarative language that allows to specify relational learning and structured output prediction problems to be solved using logical kernels. Here, we directly exploit the sequence tagging implementation of SVM-HMM to solve the SpRL problem in kLog. SVM-HMM ${ }^{5}$ uses a structural (SVM) formulation and discriminatively trains models that are isomorphic to a $k$ th-order hidden Markov model. In summary, given an observed input sequence $x=\left(x_{1} \ldots x_{l}\right)$ of feature vectors $x_{1} \ldots x_{l}$, the model predicts a tag sequence $y=\left(y_{1} \ldots y_{l}\right)$ according to the following linear discriminant function:

$$
f(x)=\arg \max _{y} w_{\mathrm{emis}}^{\top} \cdot \phi_{\mathrm{emis}}(x, y)+w_{\text {trans }}^{\top} \cdot \phi_{\text {trans }}(y)
$$

Where $w_{\text {emis }}$ and $w_{\text {trans }}$ are the emission and the transition weight vectors, respectively. The built-in NSPDK graph kernel in kLog defines the "emissions" used in the feature vector $\phi_{\mathrm{emis}}(x, y)$ used by SVM-HMM. Formulating any arbitrary structured output prediction considering the declarative representation of the output and global constraints remains as the future work.

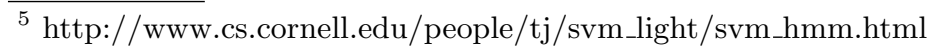



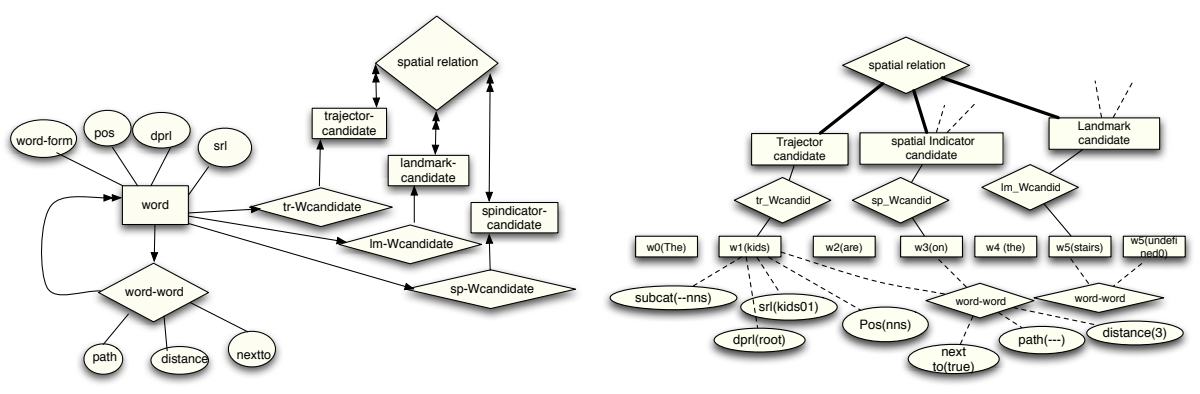

Fig. 2. (a)The E/R diagram, (b) Grounded E/R for one interpretation

\subsection{Representing SpRL in kLog}

The data model defines the entities and relationships using an $\mathrm{E} / \mathrm{R}$ diagram. Fig. 2 shows the diagram used for SpRL. The main entity of our model is a word. Word properties are obtained using the Charniak parser ${ }^{6}[2]$ and the $\mathrm{LTH}^{7}$ tool, producing linguistic features in the CoNLL-08 format ${ }^{8}$. Each word $\mathrm{w}$ is assigned an identifier $W_{-}$id and the following properties of this entity are used:

- The word form of w, represented as word(W_id,Word_form).

- The part of speech tag, represented as pos(W_id,Word_pos).

- The dependency to the syntactic head in the dependency tree, represented as dprl (W_id,Word_dprl).

- The semantic role, represented as srl(Word_id,Word_srl).

- The subcategorization of the word, sister-nodes of its parent node in the tree, represented as subcat(Word_id, Word_subcat).

In addition to the sequential relationships, a set of relational features between the words in the sentence are exploited :

- The path between the word, word (w_i, Word_form) and word ( $w_{-} j$, Word_form) in the parse tree is represented as a string with the relation path ( $\left.w_{-} i, w_{-} j, P a t h\right)$. This feature could be induced using the explicit relational representation of the parse tree. But in this work only the sequential structure is considered.

- The position of word ( $w_{-} i$,Word_form) with respect to the word ( $w_{-} j$, Word_form), represented as before $\left(w_{-} i, w_{-} j\right)$.

- The number of nodes on the pars tree's path between word ( $w_{-} i$, Word_form) and word ( $w_{-} j$,Word_form) normalized by dividing it by the number of all nodes in the parse tree, represented as distance $\left(w_{-} i, w_{-} j\right.$, Distance)

distance $=\frac{\text { \#nodes on path from word (w_i }, \text { Word_form) to word ( } w_{-} j, \text { Word_form) }}{\text { \#Nodes in the parse tree }}$

The rounded (ceiling) value of the reversed distance is used as a nominal property. Usually landmarks are relatively closer to the preposition compared to the trajectors. So this feature positively influences the learning model.

\footnotetext{
${ }^{6}$ http://www.cog.brown.edu/ mj/Software.htm

${ }^{7}$ http://barbar.cs.lth.se:8081/

${ }^{8}$ http://barcelona.research.yahoo.net/dokuwiki/doku.php?id=conll2008:format
} 
Each sentence is represented as an interpretation. This interpretation is equivalent to the unfolded (grounded) E/R diagram represented as a bi-partite graph such as Fig. 2(b). This figure shows an example with kLog's graphical representation of the relational features for the sentence "The kids are on the stairs." It shows a part of the facts that are stored in the relational tables.

Extensional tables. The given facts are stored directly in the database, in so-called extensional tables. In the above example some of these are:

word (w0, the). word (w1,kids). word (w2, are). pos (w2, vbp). pos (w3, in). pos (w4,dt). dprl(w0,nmod). dprl(w1, sbj). dprl(w2, root). dprl(w3,prd). dprl (w4, nmod). path(w1,w3, 'NNS^NP^S_VP_PP_IN'). distance (w1,w3,2). path (w5, w3, 'NNS^NP^PP_NN'). distance (w5, w3 , 3).

Intentional tables. In $\mathrm{kLog}$, logical rules can be used (as in Prolog) to define intensional relations and induce new features. For example, it is not needed to provide the next relation between adjacent words extensionally because it can be derived from the word identifiers using the following clause:

$$
\text { next (W1, W2 ): - } \operatorname{word}(W 1, \ldots) \text {, word }(W 2, \ldots) \text {, W2 is } W 1+1 \text {. }
$$

This produces the following tuples in the data base, in so-called intensional tables:

$\operatorname{next}($ w0, w1 $) \cdot \operatorname{next}($ w1, w2 $) \cdot \operatorname{next}($ w2, w3 $) \cdot \operatorname{next}($ w3, w4) $\cdot \operatorname{next}($ w4 , w5) .

Background knowledge. This is represented explicitly using logical rules in the problem specification and independent of the underlying machine learning model. In SpRL, in its binary classification formulation, to avoid an explosion in the number of examples, we limit the set of example spatial relations to a number of candidates. This is performed using a set of logical rules that we call candidate selection rules. In more details, trajectors and landmarks are mostly noun phrases and spatial indicators are often prepositions. To exploit this knowledge in $\mathrm{kLog}$, for example, the following candidate selection rules for selecting trajector candidates are provided:

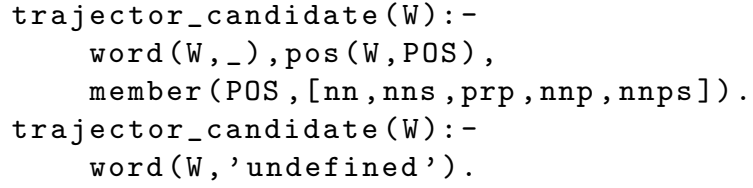

Thus, a candidate trajector is either a noun or undefined. This is applied similarly in candidate selection for landmarks and spatial indicators. The rules produce new relational entities and intentional tables. For example applying the candidate selection rules on the above sentence yields:

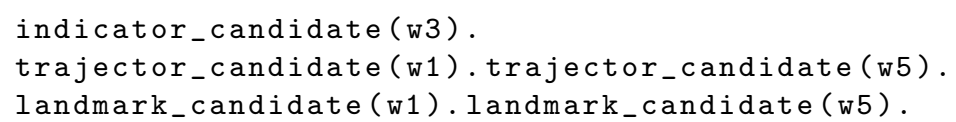

Finally, we are able to generate the relational target of the learning problem using the logical language. This provides a flexible way to represent any structured output prediction in a relational form. The target formulation is discussed in more details in the next section. In the above example, the target roles include

indicator(w3). trajector(w1). landmark(w5). 
and the target spatial relation is $\mathrm{sr}(\mathrm{w} 3, \mathrm{w} 1, \mathrm{w} 5)$.

This resembles a multi-predicate learning task [6] in which the predicates are nodes in the graphs (see. 2(b)). The graphs are turned into feature vectors using a graph kernel, which ultimately leads to a propositional learning problem in a high dimensional space.

\section{Spatial relation extraction}

In this section we discuss two different formulation of the relation extraction. The first formulation is a binary classification of triplet relations. That is, for every possible triplet of words in a sentence, hypothesize a relation between them and classify it as being spatial or not. In the second formulation, we perform relational sequence tagging and then construct the target spatial relations using a set of logical rules.

\subsection{Problem formulation I: triplet classification}

According to the problem statement, each sentence is associated with a set of true spatial relations in the form of $\operatorname{sr}(\mathrm{SI}, \mathrm{TR}, \mathrm{LM})$. A direct formulation of extracting the sr predicates includes the following processes: a) generate the possible triplets of words per sentence; b) consider each produced true triplet as a positive and each false triplet as a negative example; c) compute the features of each example; d) classify each triplet as true or false based on its computed features.

Each triplet atom is a relational node in kLog's bipartite graph. The triplet's features contain the connected nodes in its neighborhood. The neighborhood means the scope of a given radius and distance parameters of kLog's graph kernel. In this formulation, the learning problem boils down to binary classification of these atoms using the relational features and the propositionalization process. The bottleneck of this approach is the vast number of possible negative examples as compared to the positive ones. We exploit the background knowledge and also a pipelining approach to deal with this problem. Due to the use of logical representation in kLog, different settings are represented in the problem specification side, independent from the learning model.

Candidate selection. Instead of generating all combinations of arbitrary words, the candidate selection rules described in Section 2.3 are used to reduce the number of examples. Hence, the candidate triplets are produced by choosing their arguments from the previously defined candidate roles using the following rule:

sr_candidate $1(\mathrm{I}, \mathrm{T}, \mathrm{L})$ :- indicator_candidate(I),

trajector_candidate (T), landmark_candidate (L).

The predicate $\mathrm{sr}_{-}$candidate1(I,T,L) includes the positive as well as the negative examples; the positives are those for which $\operatorname{sr}(\mathrm{I}, \mathrm{T}, \mathrm{L})$ holds in the sentence and satisfy the candidate selection rules, e.g. Pos-tag restrictions. Since the coverage of candidate selection rules is not $100 \%$, some actual positive examples may not be sr_candidates 1 .

Pipelining. Another approach is to separately train three models to predict the spatial roles first and use the predicted roles to construct the examples for learning the sr predicates. In this case the positive and negative examples are produced using 


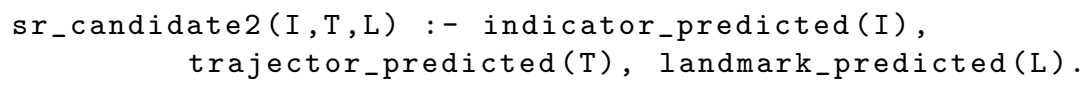

The newly introduced predicates such as indicator_predicted(I) are added to the database after the spatial role prediction. A high recall of this step is required to achieve a reasonable performance for sr prediction in the second phase. To this aim a threshold is adjusted using the precision-recall curve to increase the number of sr_candidates 2 . In this setting, the learning procedure becomes a stacked pipeline where the second stage of $\operatorname{sr}(I, T, L)$ prediction receives as examples the ground atoms

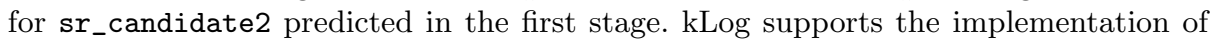
such a layered learning approach using its scripting language.

\subsection{Problem formulation II: relational sequence tagging}

To consider the sequential correlations between spatial roles, the SVM-HMM model is used. During the role prediction for each word the role of its adjacent words are considered. This model receives the input feature vectors created by kLog's graph kernel. The sequence tagging formulation is not straightforward because each word can play various roles with respect to various spatial indicators in a sentence. However, for one specific spatial indicator each word carries at most one role. This realistic assumption is used to set up relational sequence tagging. Each interpretation is presented as a sequence of word entities word (w_1, Wordform) , . ., word (w_n, Wordform) that are connected with the next $\left(w_{-} i, w_{-} j\right)$ relation and one spatial-indicator candidate is chosen as the pivot of the interpretation. The word entities are assigned a role based on the selected pivot.

A sentence with $k$ spatial indicator candidates generates $k$ examples, each having a different pivot. In this setting a new predicate role ( $w_{-} i, R_{0}$ ) is defined such that 1) role ( $w_{-} j$,spatial_indicator) is true for at most one $\left.w_{-} j ; 2\right)$ Each word $w_{i}$ is assigned the property trajector or landmark, if it carries that role with respect to the spatial indicator $w_{-} j$; and 3 ) the remaining words are assigned word (w_i,none). If the pivot is not a spatial indicator, all words in the example will have role ( $w_{-} i$,none). Target generation rules. Assuming a fixed pivot implies that pairwise relations are obtained immediately after the tagging phase. We declaratively program kLog to produce sr relations using the rules below. These rules named target generation rules. These construct the output's structure based on its predicted components.

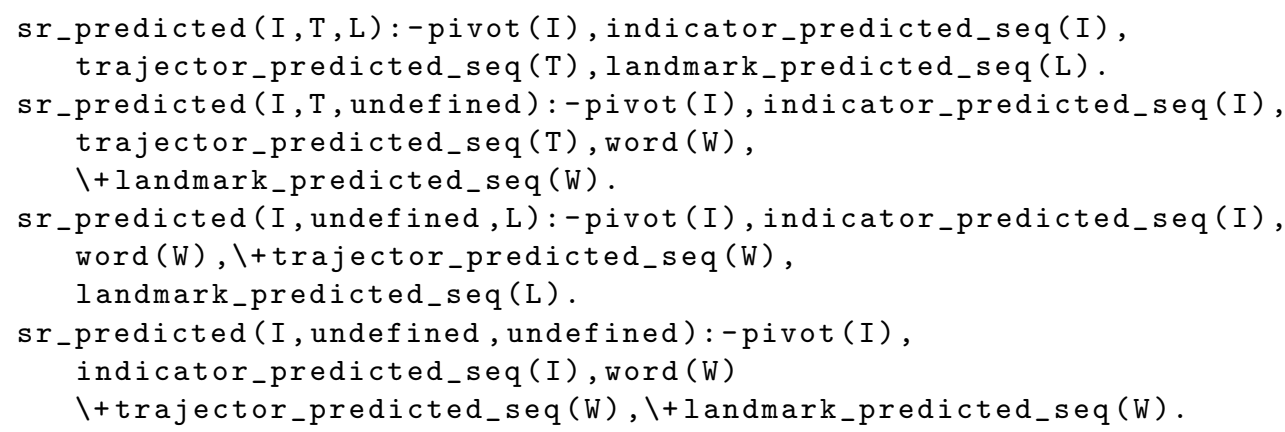

The newly introduced predicates such as indicator_predicted_seq(I) are added to the database after the sequence tagging phase. If the role of trajector or landmark is not produced then the related argument in sr is 'undefined', cf. the example in Section 2.1. In Section 4 the experimental results using SVM-HMM are presented and compared to their counterpart linear-chain CRF model. 


\section{Experiments}

In this section, the experimental setup and the results for SpRL are presented. For the described problem formulations, the following experimental questions are investigated:

1. What is the influence of candidate selection rules on spatial role and sr prediction?

2. What is the effect of pipelining spatial role learning and learning sr relations?

3. What is the influence of using SVM-HMM instead of SVM in the role learning phase of pipeline model?

4. What is the performance of the relational sequence tagging using the pivots and constructing sr's afterward by target generation rules?

5. What is the benefits of relational representation and using sequence tagging in kLog compared to CRFs?

Dataset. Our corpus ${ }^{9}$ consists of textual descriptions of 613 images in the IAPR TC12 image data set [11], denoted CLEF. It contains 1213 English sentences and 1716 corresponding spatial relations. This dataset is an extension of the one used in [15].

Evaluation. The evaluation is based on 10 -fold cross validation. For unary roles this is the classic evaluation based on true/false positive/negatives for each role. For the end-to-end evaluation of $\operatorname{sr}(\mathrm{I}, \mathrm{T}, \mathrm{L})$, the final false/true positive/negative generated triplets are counted against the ground truth sr's in the whole data base.

\subsection{Triplet classification I}

In these experiments, sets of positive and negative triplets are produced per each sentence to train a model for sr prediction. The experimental questions 1-3 are considered and to produce triplets the following options are examined: 1) Producing all possible triplets. 2) Using candidate selection rules. 3) Predicting spatial roles and pipelining.

For predicting the spatial roles in the pipeline the use of SVM and SVM-HMM is examined. We describe the spatial role prediction first because it is used in other steps.

Spatial role prediction. In the first experiment, using all words, three binary SVM models are trained independently. These form the baseline for unary predicates trajector (W), landmark (W) and indicator (W). In these models each word is an example. The total number of words in the data set is 21,308 . The number of positive roles trajectories, landmark and indicators are 1468, 1593 and 1462 respectively. The words with no role are negative examples. In spite of an imbalanced distribution of positives and negatives, the spatial role prediction is feasible with a reasonable accuracy, see Table 1 . The values up to two decimal points are significant with $95 \%$ confidence interval.

In the second experiment, using candidate selection rules (Section 2.3.) the number of negative examples is reduced to a great deal, from 21308 to 7195 for trajector/landmark candidates (about 66\%) and to 2903 for spatial indicator candidates (about $86.4 \%$ ). However, by applying these rules, on average $11 \%$ of positive roles are not covered. In this experiment, the binary SVM is used. The results indicate a statistically significant improvement in the classification of candidate words compared to using all words $(p<0.05)$. In the third experiment, the SVM is replaced by an SVM-HMM. SVM-HMM considers the sequential relationships between words in the unary spatial role prediction (trajector $(W)$, landmark $(W)$, indicator $(W)$ in separate models vs. none). Considering these correlations improved the results significantly, see Table 1.

\footnotetext{
$\overline{9}$ http://www.cs.york.ac.uk/semeval-2012/task3/
} 


\begin{tabular}{|l|l|l|l|l|l|}
\hline Examples-Model & Target predicate & F1 & Precision & Recall & \# examples \\
\hline \multirow{3}{*}{ All words-SVM } & trajector (W) & 0.68 & 0.67 & 0.68 & 21308 \\
& landmark (W) & 0.68 & 0.64 & 0.71 & 21308 \\
& indicator (W) & 0.81 & 0.81 & 0.81 & 21308 \\
\hline \multirow{3}{*}{ Cand.words-SVM } & trajector (W) & 0.72 & 0.73 & 0.72 & 7195 \\
& landmark (W) & 0.74 & 0.73 & 0.76 & 7195 \\
& indicator (W) & 0.90 & 0.90 & 0.91 & 2903 \\
\hline \multirow{3}{*}{ Cand.words-SVMHMM } & trajector (W) & 0.77 & 0.79 & 0.76 & 7195 \\
& landmark(W) & 0.86 & 0.88 & 0.85 & 7195 \\
& indicator (W) & 0.94 & 0.95 & 0.92 & 2903 \\
\hline
\end{tabular}

Table 1. Unary spatial role prediction for word entities in kLog.

Spatial relation prediction If all triplets of words are considered as possible spatial relations, the number of examples produced for sr prediction will be equal to the cube of words per sentence $(=12,346,353)$, while only 1,716 of these are positive relations. Hence, due to the huge number of negative examples, training a practical model is not feasible. By applying candidate selection rules, the number of triplets is reduced from $12,346,353$ to 190,740 . However, the disadvantage is that about 252 of positively annotated triplets are not covered and missed. This means $15 \%$ of the annotated relations that yield 1,464 positive triplets for this setting. The results of sr prediction in different experiments are presented in Table 2. The details of the settings are described in the following paragraphs.

sr_1 shows the results of triplet classification in one step by using a binary SVM backend. The candidate selection rule (i.e. the predicate sr_candidate1 defined in Section 3.1) is used to produce examples. This is the baseline of triplet classification. sr_1_1 exploits the predicted spatial role predicates in learning sr prediction model. The role predicates are added to the database and consequently to the interpretations. Then those are used as new features attached to the words for the triplet classification. Once more the sr_candidate1 is used to generate examples yet with the difference that predicted roles such as the

\begin{tabular}{|l|l|l|l|}
\hline Target predicate & F1 & Precision & Recall \\
\hline sr_1(W, W', W" ) & 0.52 & 0.50 & 0.55 \\
sr_1_1(W,W', W" $)$ & 0.54 & 0.50 & 0.58 \\
\hline sr_2(W,W', W" ) & 0.60 & 0.48 & 0.80 \\
sr_2_1(W,W', W' $)$ & 0.50 & 0.48 & 0.52 \\
\hline sr_3(W, W' , W" ) & 0.71 & 0.68 & 0.74 \\
sr_3_1(W,W', W' $)$ & 0.58 & 0.68 & 0.50 \\
\hline
\end{tabular}
Table 2. triplet relation classification the results of sr_1_1 compared to sr_1 in Table 2 by $2 \% \pm 1 \%$ with a $95 \%$ confidence interval for the F-measure.

sr_2 presents the performance of the relation classification in the pipeline model. In this experiment the first step of the pipeline uses the candidate selection rules and the SVM. Given the recall of spatial role prediction in Table 1, using these assignments for selecting candidates leads to a large miss about $40 \%$ of the true positives. However, we did stacking over the role classification and triplet classification steps. The sr_candidate2 predicate is used to generate examples for the second layer, see Section 3.1. The results of stacking are presented in $\mathrm{sr}_{-} 2$ line in Table 2 . These indicate a $6 \%$ improve in the F-measure compared to the one step relation classification. The missed positives are ignored in this line of reported results which implies a perfect system is needed in the first phase of pipelining to obtain this overall performance.

sr_2_1 evaluates the whole pipeline system starting from the input sentences. This end-to-end evaluation indicates a decrease in performance compared to sr_1. This is due to the propagated errors from the spatial role prediction step. 
sr_3 aims to improve the performance of the pipeline by reducing the errors of the first step. It uses the stronger model SVM-HMM for spatial role prediction. The triplet classification is performed using the same binary SVM. Due to the improvement made in the first stage, the triplet classification in the second phase significantly improved

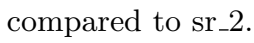

sr_3_1 examines the end-to-end evaluation of the pipeline model of sr_3. Compared to the counterpart of this experiment $\mathrm{sr}_{\_} 2 \_1$, an $8 \% \pm 0$ improvement is observed. This model performs $4 \% \pm 0$ better than sr_1_1 too, (with a $95 \%$ confidence interval).

An overall comparison according to the results in Table 2 indicates that the pipeline system that uses the candidate selection rules along with SVM-HMM for the role prediction and then SVM for the second phase of triple classification is the best model in the above experiments. This answers the questions 1-3.

\subsection{Sequence tagging for relation extraction II}

The goal of this section is to investigate the experimental questions 4-5. The main difference of these experiments compared to the last ones is that the direct target of the learning model is relational sequence tagging. This is performed using the sequence tagging technique of SVM-HMM [19] plugged into kLog. This type of modeling for SpRL is described in Section 3.2. The setting is without candidates selection. The number of examples in this experiment is equal to the number of candidate prepositions; that is about 2903 tagged sequences. The predicate $\mathrm{role}_{\mathrm{e}}\left(\mathrm{w}_{-} \mathrm{j}, R\right)$ is predicted by the sequence tagger. This predicate assigns a relational spatial role $\mathrm{R}$ to each word with respect to a predefined pivot in each sequence. Spatial triplets derived by a set of rules and the predicate $\mathrm{sr}_{-}$predicted $(\mathrm{I}, \mathrm{T}, \mathrm{L})$ are produced directly based on the predicted relational roles in the sequence. Producing the interpretations in kLog for this experiment is described in Section 3.2.

\begin{tabular}{|l|l|l|l|l|l|l|l|l|}
\hline \multicolumn{4}{|c|}{ kLog (SVM-HMM) } & & \multicolumn{4}{c|}{ Linear-chain CRF } \\
\cline { 1 - 7 } Target predicate & F1 & Precision & Recall & & Target predicate & F1 & Precision & Recall \\
\hline trajector (W) & 0.71 & 0.75 & 0.68 & & trajector (W) & 0.79 & 0.83 & 0.76 \\
landmark (W) & 0.87 & 0.89 & 0.85 & & landmark (W) & 0.88 & 0.92 & 0.84 \\
indicator (W) & 0.93 & 0.92 & 0.93 & & indicator (W) & 0.94 & 0.92 & 0.96 \\
sr(W,W',W") & 0.60 & 0.57 & 0.63 & & sr (W,W', W") & 0.65 & 0.65 & 0.65 \\
\hline
\end{tabular}

Table 3. Results of CRF, SVM-HMM, 10-fold cross-validation

Even by ignoring the missed positives in candidate selection of the triplet classifier, relational sequence tagging performs best for this task, see Table 3 . In Table 3, we present the experimental results of our previous work [15] using CRFs too. The current results are not better but fairly comparative to the results of CRFs. Given the flexibility that is provided by $\mathrm{kLog}$ for declaratively representing the problem and considering the use of background knowledge these results are promising. The future improvements will be interfacing flexible structured output prediction in kLog.

\subsection{Experimental analysis}

Comparing the two main sets of experiments, indicates the relational sequence tagging assuming a pivot is the best model which is implemented by both CRFs and kLog. Although CRFs are slightly outperforming, kLog provides a declarative language to 
present the model, including generating examples for train/test from the database, performing relational feature engineering and generating the structure of the output, independent from the underlying learning model. These are again promising answers to the question 5 .

The performance of triplet classification was low due to the huge number of negative examples, even in the candidate selection setting. Pipelining and stacking have a low performance due to the error propagation between the spatial role prediction and the sr prediction phase. However using SVM-HMM in the first step of the pipeline yielded better results.

In the following paragraphs we provide a brief analysis on the relational representation of the features and the flexible way that kLog deals with them in the graphicalization process and propositionalization. A more extensive analysis for the influence of the linguistic features and the errors is given in [15] within the propositional modeling.

Feature analysis We performed an experimental analysis of the features by gradually incorporating them in the training phase. In the triplet classification, even after using candidates a huge number of negatives remained. Therefore, less dense graphs are used for the sake of efficiency. In these experiments the path and distance features are not used, but only the "next" relation connects the word entities in each interpretation. Path and distance are used in the sequence tagging approach.

In the process of graphicalization and generating the propositionalized features in $\mathrm{kLog}$, one can easily set two parameters called radius and distance. These guide the graph kernel in selecting the pairwise subgraphs for computing the kernel matrix. Intuitively this allows us to indicate the size of the relational features and an upper bound for the distance in the graph to be considered in feature generation. We observed that the "next" relation has a strong increasing influence in classification of the roles and consequently in classification of sr relations. This improvement was $6 \%$ for trajectors, $3 \%$ for indicators and $2 \%$ for landmarks. However increasing the distance and radius for generating relational features does not always increase the performance and obviously these parameters can be optimized by using a validation set. One other observation was that involving the predicted trajector roles improved the prediction of landmarks by $3 \%$ and the mutual influence of landmarks was also positive.

Error analysis We provide an extensive description of the error types for spatial role labeling in [15]. Related to the present work, one main source of error in both triplet classification and sequence tagging is the relatively large number of negatives compared to the positives. This leads to a bias towards no role assignment to the words in multi-class sequence tagging and also to classifying the triplets as negative in the triplet classification. However, this issue is more problematic in triplet classification and dramatically decreases the performance. We performed some experiments using a random selection of negatives to create a balanced data set. In these experiments a 10fold-cross validation over the same data shows a very high precision and recall. However in a realistic setting in which all the possible negatives should be tested, even a $99 \%$ accuracy on the negatives introduces a large number of false positives and decreases the precision sharply. One possible future solution to this problem is to select a subset of negatives. Our future plan is to provide a flexible structured output prediction in kLog and consider the most violated constraints in the max-margin optimization process [19] in the training phase. We will interface the arbitrary structured output prediction with a declarative representation in $\mathrm{kLog}$. 
Moreover, the error analysis indicates the importance of the lexical features and also the necessity of a large annotated dataset to increase the performance. A solution to this problem is to use linguistic resources, which are often available in a logical representation [5], and which can be exploited naturally in kLog. The expansion of lexical information allows to improve the cross domain portability of the model too.

\section{Conclusion}

We formulated the spatial role labeling task as a relational learning problem in kLog. $\mathrm{kLog}$ allows using a flexible logical representation of the data and knowledge compared to the propositional models. The relational features can be defined in Prolog and are exploited by a graph kernel. In this way, kLog provides a flexible platform to efficiently experiment with various relational formulations of the problem.

Our first formulation was based on triplet classification, where we classify the spatial relations that connect the three main spatial roles of trajector, landmark and spatial-indicator. The main difficulty of this setting is the huge number of negative triplets as compared to the positive ones. Using background knowledge in the form of candidate selection rules helped to reduce the number of candidate words and consequently to reduce the number of negative triplets. This made the sr relation prediction feasible though the performance was lower than some other settings.

For the same formulation of triplet classification we used a pipeline. In this setting the spatial roles are predicted and used as the candidates for constructing the triplets. The triplet classification is performed in a second phase. The end-to-end evaluation for prediction of sr relations, had a low performance due to the error propagation from the first step. However, using SVM-HMM for the first phase of the pipeline provided the best results in this first formulation.

The second formulation employed a relational sequence tagging model based on the pivots. In this model we predicted the roles of the words based on the candidate pivots and then induced the spatial relations based on the target generation rules. We employed a SVM-HMM model in kLog. This led to improved results as compared to the triplet classification. The results with this approach were comparable to experiments using CRFs. However, the logical representation of kLog provides the required flexibility for specifying the relational features and the structure of the output declaratively.

Our two main future directions are: a) Interfacing kLog's declarative representation to arbitrary structured output prediction. This will resolve the problem of huge numbers of negatives in the sr relation prediction moreover we can consider the correlations between the predicted triples per each sentence. b) Extending the SpRL task and mapping the extracted spatial relations to a formal spatial calculus and spatial ontologies. The formal spatial representation will make spatial reasoning based on linguistic descriptions, feasible [12]. kLog's logical language and relational kernel-based learning provides the necessary flexibility to achieve this goal.

\section{References}

1. J. A. Bateman. Language and space: a two-level semantic approach based on principles of ontological engineering. International Journal of Speech Technology, 13(1):29-48, 2010. 
2. E. Charniak and M. Johnson. Coarse-to-fine n-best parsing and maxent discriminative reranking. In Proceedings of the 43rd Annual Meeting on Association for Computational Linguistics, ACL '05, pages 173-180, 2005.

3. A. G. Cohn and J. Renz. Qualitative spatial representation and reasoning. In Handbook of Knowledge Representation, volume 3 of Foundations of Artificial Intelligence, pages 551 - 596. Elsevier, 2008.

4. F. Costa and K. De Grave. Fast neighborhood subgraph pairwise distance kernel. In Proceeding of 27th ICML, 2010.

5. J. Cussens. Issues in learning language in logic. In Antonis Kakas and Fariba Sadri, editors, Computational Logic: Logic Programming and Beyond, volume 2408 of $L N C S$, pages 171-177. 2002.

6. L. De Raedt, P. Frasconi, K. Kersting, and S. Muggleton, editors. Probabilistic Inductive Logic Programming, volume 4911 of LNCS. 2008.

7. P. Domingos and M. Richardson. Markov logic: A unifying framework for. In ICML-2004 Workshop on Statistical Relational Learning and its Connections to Other Fields, pages 49-54, 2004.

8. P. Frasconi, Costa F., De Raedt L., and De Grave K. klog - a language for logical and relational learning with kernels, 2011.

9. A. Galton. Spatial and temporal knowledge representation. Journal of Earth Science Informatics, 2(3):169-187, 2009.

10. H. Garcia-Molina, J. D. Ullman, and J. Widom. Database Systems: The Complete Book. Prentice Hall Press, 2 edition, 2008.

11. M. Grubinger, P. Clough, Henning Müller, and Thomas Deselaers. The IAPR benchmark: A new evaluation resource for visual information systems. In International Conference on Language Resources and Evaluation (LREC), 2006.

12. P. Kordjamshidi, J. Hois, M. van Otterlo, and M.-F. Moens. Machine learning for interpretation of spatial natural language in terms of QSR. Poster at the 10th International Conference on Spatial Information Theory (COSIT'11), 2011.

13. P. Kordjamshidi, M. van Otterlo, and M. F. Moens. From language towards formal spatial calculi. In Workshop on Computational Models of Spatial Language Interpretation (CoSLI 2010, at Spatial Cognition 2010), 2010.

14. P. Kordjamshidi, M. van Otterlo, and M. F. Moens. Spatial role labeling: Task definition and annotation scheme. In LREC'10, 2010.

15. P. Kordjamshidi, M. Van Otterlo, and M.F. Moens. Spatial role labeling: Towards extraction of spatial relations from natural language. ACM Trans. Speech Lang. Process., 8:1-36, December 2011.

16. A. McCallum, K. Schultz, and S. Singh. Factorie: Probabilistic programming via imperatively defined factor graphs. In NIPS, 2009.

17. Mihai Surdeanu, Lluis Marquez, Xavier Carreras, and Pere R. Comas. Combination strategies for semantic role labeling. Journal of Artificial Intelligence Research, pages 105-151, June 2007.

18. D. A. Tappan. Knowledge-Based Spatial Reasoning for Automated Scene Generation from Text Descriptions. PhD thesis, 2004.

19. I. Tsochantaridis, T. Joachims, T. Hofmann, and Y. Altun. Large margin methods for structured and interdependent output variables. Journal of Machine Learning Research, 6(2):1453-1484, 2006.

20. G. Wachman and R. Khardon. Learning from interpretations: a rooted kernel for ordered hypergraphs. In ICML, volume 227, pages 943-950, 2007.

21. J. Zlatevl. Spatial semantics. In Hubert Cuyckens and Dirk Geeraerts (eds.) The Oxford Handbook of Cognitive Linguistics, Chapter 13, pages 318-350, 2007. 